In der Volksheilkunde ist die naturheilkundliche Behandlung von Wunden, entzündeter Haut und anderen dermatologischen Erkrankungen weit verbreitet. Heilpflanzen haben in der Volksmedizin für die Behandlung sowohl von akuten als auch von chronischen Wunden eine grosse Bedeutung. In der schulmedizinischen Behandlung hingegen wurden erst in den letzten Jahren teilweise wieder Heilpflanzen-Extrakte bzw. Fertigpräparate mit pflanzlichen Wirkstoffen eingesetzt. Neben positiven Fallberichten gibt es für einige in der Wundheilkunde bekannte und erfolgreiche Heilpflanzen positive Monografien der Kommission E. Klinische Studien mit Heilpflanzen-Anwendungen für die Wundversorgung sind jedoch äusserst selten. Betrachtet man die Problematik vorschneller Glukokortikoid- und Antibiotika-Gaben, wird deutlich, dass das grosse Potenzial phytotherapeutischer Anwendungen gerade in der Haut- und Wundpflege genutzt werden sollte.

\section{Die Apotheke der Natur}

«Alle Wiesen und Matten, alle Berge und Hügel sind Apotheken!» (Paracelsus [1])

Die Naturapotheke aus Wald, Wiese und Garten stellt viele Heilpflanzen für die Sofortanwendung und erste Hilfe, aber auch für die Behandlung von schwer heilenden Wunden oder grösseren Verletzungen bereit. Wie das eingangs genannte, bekannte Zitat von Paracelsus besagt, wachsen Heil- pflanzen zwischen Gartentor, Waldspaziergang und Blumenwiese. Selbstverständlich ist bei ernsthaften Verletzungen eine ärztliche Abklärung notwendig.

Spezifische Heilpflanzen eignen sich besonders:

- bei Bagatellverletzungen,

- zur Sofortbehandlung, wenn man unterwegs ist, und als Vorbehandlung, bevor ein Arzt aufgesucht werden kann, und

- bei chronischen, schlecht heilenden Wunden (für die in der Schulmedizin keine weitere Behandlung mehr bekannt ist).

In Tabelle 1 werden einige bekannte und weit verbreitete Heilpflanzen aus der Naturapotheke vorgestellt [26].

\section{Die Haut}

Als grösstes Sinnesorgan des Menschen schützt die Haut den Menschen vor äusseren Einflüssen und ist entscheidend für seine Erscheinung und sein Wohlbefinden. Als äusserst sensibles Funktionssystem ist die Haut die sichtbarste Verbindung von seelischen Prozessen und organischen Strukturen.

Zu den Aufgaben der Haut zählen:

- Schutz-, Abwehr und Immunfunktion;

- Speicherfähigkeit;

- Wärmeregulation;

- Hautatmung;

- Ausscheidungs- und Entgiftungsfähigkeit;

- Resorption/Aufnahme;

- Sinneswahrnehmung;
- Kommunikation;

- Funktion als diagnostisches Hilfsmittel.

Ihre vielfältigen Aufgaben kann die Haut nur erfüllen, wenn sie intakt und funktionsfähig ist. Auch deshalb ist die Behandlung von akuten und chronischen Wunden sehr wichtig.

Bei Hauterkrankungen sowie schlecht heilenden, chronischen Wunden müssen verschiedene Ursachen und Grunderkrankungen abgeklärt und ursächlich behandelt werden (Diabetes, chronisch-venöse Insuffizienz, Entzündungen der Gefässe, Durchblutungsstörungen usw.). Trotzdem hat eine gute Wundversorgung eine grosse Bedeutung, damit die Stadien der Wundheilung - Exsudation, Granulation und Epithelisierung - physiologisch sinnvoll ablaufen.

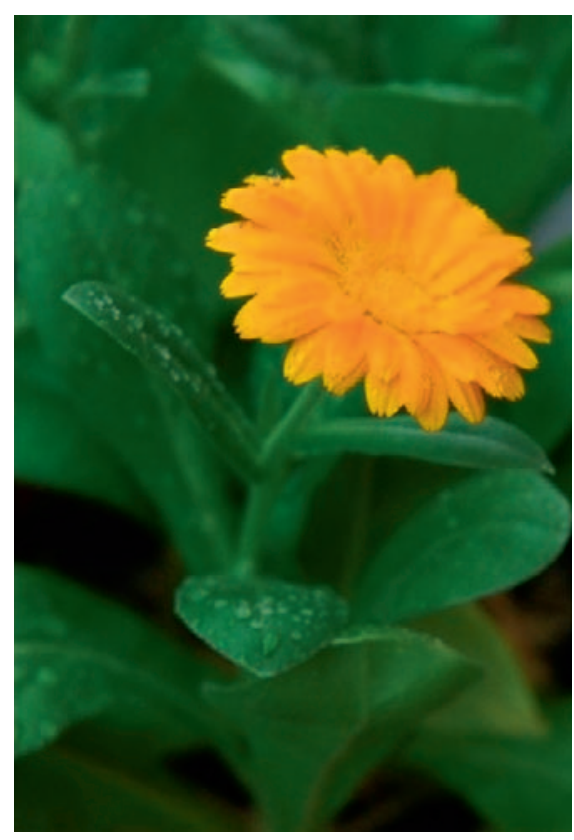

Abb. 1. Ringelblume (Calendula officinalis).

\section{KARGER}

(C) 2015 S. Karger GmbH, Freiburg

Fax +497614520714 
Tab. 1. Bekannte und weit verbreitete Heilpflanzen aus der Naturapotheke

\begin{tabular}{|c|c|}
\hline $\begin{array}{l}\text { Beinwell, Wallwurz (Symphytum } \\
\text { officinale) }\end{array}$ & $\begin{array}{l}\text { Wundumschläge mit frisch gequetschten Blättern oder frisch } \\
\text { geriebener Wurzel (Symphyti folium, radix), hoher Allantoin- } \\
\text { gehalt }\end{array}$ \\
\hline Blutwurz (Potentilla erecta) & $\begin{array}{l}\text { blutstillende und adstringierende Wirkung der Wurzelabkochung } \\
\text { (Tormentillae rhizoma) }\end{array}$ \\
\hline Eiche (Quercus robur) & $\begin{array}{l}\text { Abkochung der Rinde (Quercus cortex) bei nässenden Ekzemen, } \\
\text { Frostbeulen, Hämorrhoiden und Hautentzündungen }\end{array}$ \\
\hline Gänseblümchen (Bellis perennis) & $\begin{array}{l}\text { Auflage der frisch zerdrückten Blätter (Bellis perennis folium) } \\
\text { bei Hautschürfungen, kleinen Wunden und Insektenstichen } \\
\text { (sehr beliebt als «Pflaster» bei Kindern) }\end{array}$ \\
\hline $\begin{array}{l}\text { Hamamelis, Zaubernuss } \\
\qquad \text { (Hamamelis virginiana) }\end{array}$ & siehe Text \\
\hline $\begin{array}{l}\text { Hirtentäschchen } \\
\text { (Capsella bursa-pastoris) }\end{array}$ & $\begin{array}{l}\text { das blühende Kraut (Bursae pastoris herba) wirkt blutstillend } \\
\text { bei oberflächlich blutenden Wunden, auch bei Nasenbluten }\end{array}$ \\
\hline $\begin{array}{l}\text { Johanniskraut } \\
\text { (Hypericum perforatum) }\end{array}$ & siehe Text \\
\hline Kohl (Brassica oleracea) & $\begin{array}{l}\text { frische Blätter (Brassicae folium), von der dicken Mittelrippe } \\
\text { befreit und mit dem Wallholz gequetscht, als Auflage bei } \\
\text { entzündeten, eitrigen Wunden }\end{array}$ \\
\hline $\begin{array}{l}\text { Malve (Malva neglecta, } \\
\text { Malva sylvestris) }\end{array}$ & $\begin{array}{l}\text { Tee aus Blüten oder Blättern (Malvae flos, folium) als Auflage } \\
\text { oder Bad bei Verletzungen, Juckreiz, eitrigen Wunden, Abszessen, } \\
\text { Furunkeln }\end{array}$ \\
\hline Ringelblume (Calendula officinalis) & siehe Text \\
\hline Sanikel (Sanicula europaea) & Wundauflage mit dem Blätterauszug, enthält Allantoin \\
\hline Schafgarbe (Achillea millefolium) & siehe Text \\
\hline Spitzwegerich (Plantago lanceolata) & siehe Text \\
\hline Stiefmütterchen (Viola tricolor) & $\begin{array}{l}\text { Teeabkochung des Krauts (Violae tricoloris herba) bei Haut- } \\
\text { entzündungen, Schürfwunden }\end{array}$ \\
\hline $\begin{array}{l}\text { Stinkender Storchenschnabel } \\
\text { (Geranium robertianum) }\end{array}$ & $\begin{array}{l}\text { Auflage der frischen Blätter (Geranii folium) auf Wunden } \\
\text { wirkt antiseptisch und fördert die Vernarbung; der Blutrote } \\
\text { Storchenschnabel (Geranium sanguineum) kann gleichermassen } \\
\text { verwendet werden }\end{array}$ \\
\hline Walnuss (Juglans regia) & $\begin{array}{l}\text { Umschläge und Bäder mit der Abkochung der Blätter (Juglandis } \\
\text { folium) bei chronischen Ekzemen, Milchschorf und entzündeter } \\
\text { Haut }\end{array}$ \\
\hline Wundklee (Anthyllis vulnera) & $\begin{array}{l}\text { zerquetschte Blätter oder Blüten (Anthyllidis folium, flos) bzw. } \\
\text { deren Abkochung als Wundauflage und -umschlag }\end{array}$ \\
\hline
\end{tabular}

\section{Phytotherapeutische Anwendung} in der Wundversorgung

Nachfolgend werden einige Heilpflanzen ausführlicher vorgestellt, die sich in der Wundpflege bewährt haben und in einer modernen Wundheilkunde ihren Platz haben (sollen) [1-6].

\section{Ringelblume (Calendula officinalis)}

Die Ringelblume (Abb. 1) ziert viele Gärten vom Frühlingsanfang bis in den Spätherbst. Sie wendet sich mit ihren leuchtend orangen Blüten der Sonne zu und wird deshalb auch «Son- nenbraut» genannt. Der Name «Ringelblume» bezieht sich auf die Samen, die sich in den verblühten Blütenköpfen ringeln und seltsam archaisch anmuten. Der Name «Calendula» kommt aus dem Lateinischen («Calendis», dt. «Monate») und rührt wahrscheinlich daher, dass die Ringelblume die Sonnenbewegung angibt und mit der Sonne «auf- und untergeht». Eine andere Erklärung für den Namen «Calendula» besagt, dass die Ringelblume über den ganzen Kalender (ausser in den kalten Wintermonaten das ganze Jahr lang) blüht.

C. officinalis ist eine der bekanntesten Wundheil- und Hautpflanzen und sehr gut verträglich - trotz ihrer Zugehörigkeit zur Familie der Korbblütler (Compositae), deren andere Vertreter bekanntermassen bei einer allergischen Disposition nicht zu empfehlen sind (Korbblütler-Allergie). Die Fähigkeit der Ringelblume, Wunden schnell und komplikationslos abheilen zu lassen sowie (über-)empfindliche Haut zu pflegen und stärken, erklärt, warum die Verwendung von $\mathrm{Ca}$ lendula so beliebt ist. RingelblütenAnwendungen fördern die Wundheilung, Granulation und Fibrinbildung und wirken entzündungshemmend, lymphflussfördernd, antibakteriell (Staphylococcus aureus), virustatisch, fungistatisch (Trichomonaden), antiödematös, immunstimulierend sowie aufgrund der Triterpensaponine zytotoxisch gegenüber verschiedenen Typen von Tumorzellen [2].

Verwendet werden die Blüten (Calendulae flos), wobei im Fachhandel meist «nur» die Zungenblüten vertrieben werden - auch wenn im Kelch und in den Röhrenblüten etwa zehnmal mehr ätherische Öle und auch Allantoin vorkommen.

Die wichtigsten Inhaltsstoffe sind Triterpensaponine, Flavonoide, Carotinoide, Triterpenalkohole, Cumarine, Polysaccharide und ätherische Öle $[2,4]$.

\section{Johanniskraut (Hypericum perforatum)}

Der Ölauszug aus $H$. perforatum (Abb. 2), Johannisöl oder Rotöl (Oleum Hyperici) genannt, ist ein hervorragendes Wundheilmittel und wurde in Ausgabe 1/2015 der SchweizerISCHEN ZEITSCHRIFT FÜR GANZHEITSMEDIZIN ausführlich vorgestellt [7].

Wichtig für den Einsatz des Johannisöls in der Wundbehandlung sind die adstringierend wirkenden Gerbstoffe sowie das Hyperforin, das antibakteriell, viruzid und antimykotisch wirkt. Rotöl fördert die Wundheilung und unterstützt eine gute Narbenbildung. 


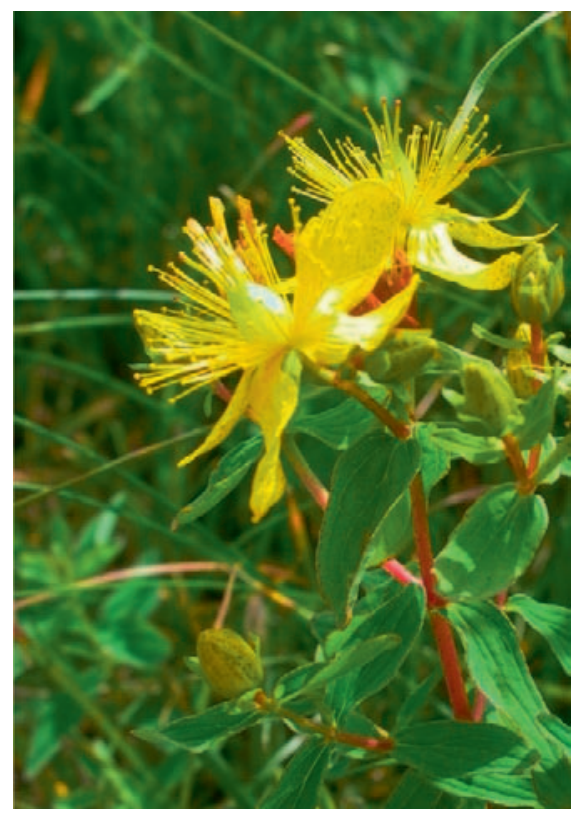

Abb. 2. Johanniskraut (Hypericum perforatum).

\section{Neembaum (Azadirachta indica)}

Der Neembaum wächst in Indien, Burma und Pakistan und wird dort als Allheil-Baum verehrt. Heute wird der Neembaum auch in tropischen und subtropischen Gegenden Afrikas, Australiens und Amerikas kultiviert. Sein Name «Neem» bedeutet auf Sanskrit «Heilspender». Rinde, Holz, Blätter, Wurzel und Früchte werden traditionell gegen diverse Leiden von Mensch und Tier eingesetzt. Der Baum hat meist eine Höhe von 15-30 m, einen kurzen Stamm sowie unpaarig gefiederte Blätter und gehört zur Familie der Mahagonigewächse (Meliaceae). Sein Holz verfärbt sich rot, wenn es mit Luft in Berührung kommt.

In der modernen Wundpflege wird vor allem das Öl der Kerne verwendet. Die Inhaltsstoffe sind bis heute nicht vollständig erforscht. Die Fettsäuren des Neemöls (Oleum Azadirachtae) wirken antimikrobiell. Besonders wichtig ist das Azadirachtin, dem die Funktion eines Insektizids zukommt. Weitere Inhaltsstoffe sind Samannin, Meliantriol, Nimbin und Nimbidin $[2,6]$.

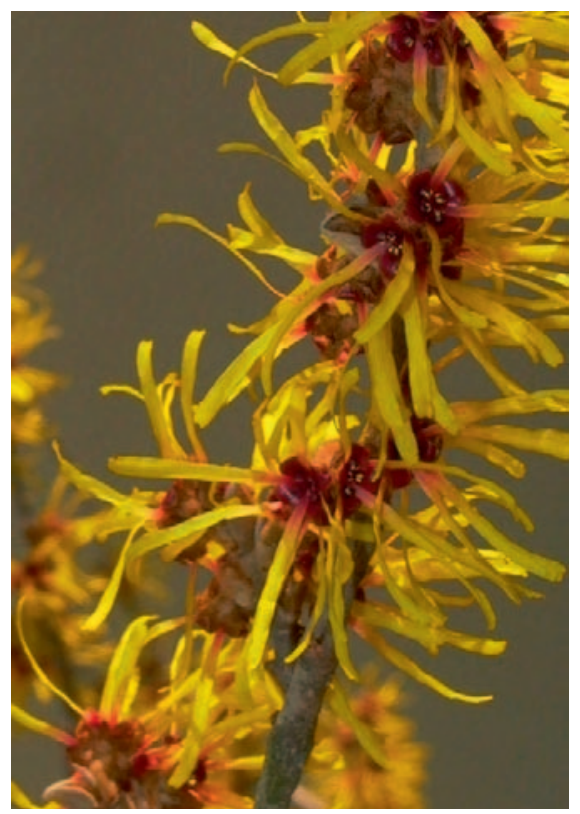

Abb. 3. Hamamelis/Zaubernuss (Hamamelis virginiana).

Seit 2010 ist ein Wundheilmittel aus der Kombination von Neemöl (Oleum Azadirachtae), Johannisöl (Oleum Hyperici) und Olivenöl (Oleum oleae) europaweit zugelassen, das bei schweren Wunden die Granulation und die Wundheilungsprozesse in Gang zu versetzen mag. Am Universitätsspital Zürich wurde es von Dr. med. Severin Läuchli, Facharzt für Dermatologie, mit Erfolg eingesetzt. Fachärzte an verschiedenen Schweizer Wundambulatorien testeten die Anwendung an über 100 Patientinnen und Patienten mit verschiedenen chronischen und akuten Wunden. Ausgewertet wurden in der Studie Wundverschluss (Komplett-/Teilverschluss), Dauer der Behandlung, Granulationsentwicklung, Schmerzen, Gewebebildung in der Wundumgebung, Handhabung für das medizinische Personal sowie die Nebenwirkungen. Die publizierte Studie kommt zu dem Ergebnis, dass sich die spezielle Kombination von Neemund Johannisöl zur Behandlung einer Vielzahl von akuten und chronischen Wunden eignet [8]. Heute wird das Wundheilprodukt («1 Primary Wound Dressing $\left.{ }^{\circledR} »\right)$ in Spitälern, Pflegeheimen und ärztlichen Praxen eingesetzt. Auch ist ein OTC-Präparat in Spray- form zur Behandlung von Schürfund Schnittwunden, Verbrennungen, Hautdefekten durch Hauterkrankungen sowie schlecht heilenden Wunden erhältlich («Wunde»). Es wirkt antimikrobiell, fördert die Wundheilung, unterstützt und verbessert die Narbenbildung und ermöglicht einen schmerzarmen Verbandwechsel.

\section{Hamamelis/Zaubernuss (Hamamelis virginiana)}

Die goldgelben Hamamelis-Blüten (Abb. 3) blühen im Winter (vor allem im November und Dezember) und leuchten auffällig und antizyklisch in der eintönigen Wintervegetation unserer Breitengrade. Möglicherweise wird Hamamelis deshalb Zaubernuss genannt. Eine andere Namenserklärung bezieht sich auf die Anwendung der Zaubernusszweige als Wünschelruten (ähnlich wie Haselzweige). Hamamelis kommt aus dem Griechischen («hamatos melos»), wird mit «hakiger Apfel» übersetzt und weist auf die Form der kleinen Früchte hin. «Virginiana» gibt die Herkunft der Heilpflanze (Virginia/USA) an. Die Zaubernuss ist ein Busch-Strauch ohne wirkliche Stammbildung und gehört zur Familie der Zaubernussgewächse (Hamamelidaceae). Die Blätter (Hamamelidis folium) enthalten 3-8\% Gerbstoffe, Proanthocyanidine, Flavonoide, Kaffeesäure-Derivate sowie ätherische Öle. In der Rinde (Hamamelidis cortex) finden sich 8-12\% freie Gallussäuren, Ellagitannin, Catechingerbstoffe sowie wenig ätherische Öle [2, 4].

Für Anwendungen mit Hamamelidis folium und cortex wurden adstringende, entzündungshemmende, blutstillende, wundheilungsfördernde, vasokonstriktorische, leicht oberflächenanästhesierende sowie juckreizstillende Wirkungen beobachtet und dokumentiert. Ausserdem verdichtet Zaubernuss das Gewebe, hemmt die Sekretion sowie Kapillarpermeabilität und schützt die Hautzellen vor oxidativen Schäden. 
Die Wirksamkeit von wässrigen und ethanolisch-wässrigen Arzneimitteln beruht mehrheitlich auf dem adstringierenden Effekt der Gerbstoffe. Wasserdampfdestillate hingegen sind gerbstofffrei. Ihre Heilkraft wird auf die ätherischen Öle zurückgeführt. Bewährt hat sich die Anwendung von wässrigen und ethanolisch-wässrigen Arzneimitteln und Wasserdampfdestillaten im Wechsel [2].

Ursel Bühring schreibt über Hamamelis: «Zaubernuss gilt auch in der Wundheilung als Zauberpflanze: In Studien lagen Therapieerfolge bei Neurodermitis vielfach im Bereich leichter Glukokortikoidgaben» [2].

\section{Hängebirke (Betula pendula)/ Moorbirke (Betula pubescens)}

Die Birke, Symbol des Frühlings, der Jugendlichkeit, der Flexibilität und der Beweglichkeit, wird auch «Nierenbaum» genannt. Birkenblätter (Betulae folium) werden seit jeher innerlich zur Behandlung von Hautleiden verabreicht. An der SMGP-Tagung 2012 stellte Prof. Dr. med. Dipl. Biol. Christoph M. Schempp, Universitäts-Hautklinik Freiburg i.Br., die Anwendung von Birkenrinde (Betulae cortex) bzw. des weissen Korks der Rinde in der Wundmedizin vor. Birkenkork enthält als Hauptwirkstoff Betulin, ein pentazyklisches Triterpen. Der Extrakt aus Birkenkork ist ausserdem ein Oleogel-Bilder, was die Herstellung von Cremen ohne Emulgatoren und Konservierungsstoffe ermöglicht. Gerade bei Hautpatientinnen und -patienten mit multiplen Kontaktallergien ist dies von grosser Bedeutung [8].

\section{Edelweiss (Leontopodium alpinum)}

Das Edelweiss (Abb. 4), auch Löwenfüsschen, Ewigweiss, Alpen-, Gletscher- und Schneestern oder Königin der Alpenblumen genannt, kommt ursprünglich aus der asiati-

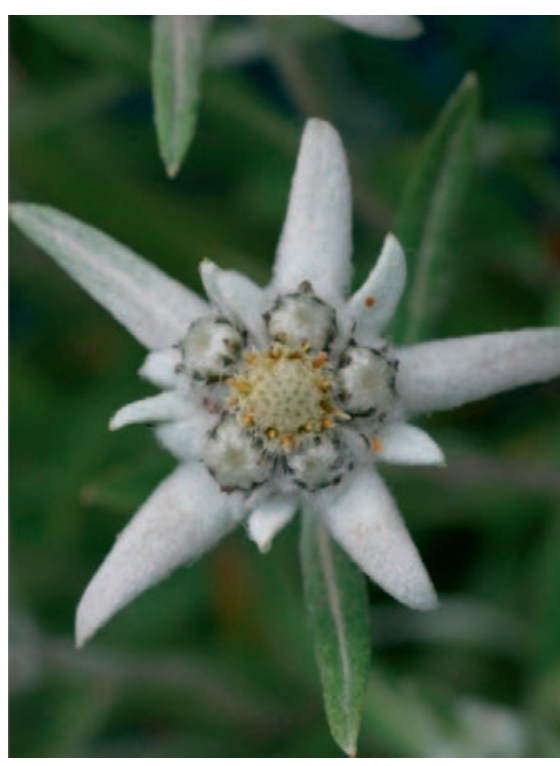

Abb. 4. Edelweiss (Leontopodium alpinum).

schen Steppe (vor allem Mongolei) sowie aus dem Himalaya. Die verehrte «weiss-pelzige Alpenschönheit» aus der Familie der Korbblütler (Asteraceae) ist heute im Alpenraum in Höhen zwischen 1700 und 3000 Meter über dem Meer heimisch. Die tiefen Faserwurzeln und die filzige Bedeckung von Blüten und Blättern schützen das Edelweiss vor Kälte, Schnee und Eis, Dürre, austrocknenden Winden und starker Sonneneinstrahlung - es ist sehr gut an die extremen klimatischen Verhältnisse in den asiatischen Steppen und den europäischen Alpenregionen angepasst.

Die spinnwebenartige Behaarung betrifft die ganze Pflanze und ist das Hauptmerkmal der geschützten Alpenblume, die in bis zu 40 Arten vertreten ist. Die weissen, filzigen Hochblätter, oft irrtümlich als Blüte angesehen, umhüllen die gelblichen Röhrenblüten, den eigentlichen Blütenstand.

In der Volksheilkunde wird das Edelweiss als haltbare Blume bis heute als Symbol der Ewigkeit in den Stuben der Bergbevölkerung aufgehängt («ewige Blume»). Neben der Wundpflege wurde es - mit Milch und Honig gekocht - als beliebtes Bauchwehmittel verwendet. Darum trägt die

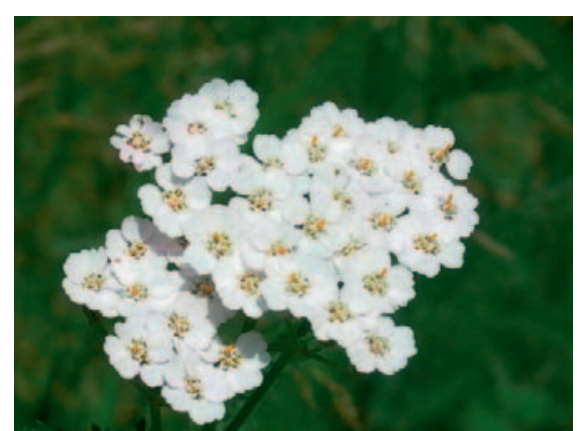

Abb. 5. Schafgarbe (Achillea millefolium).

geschützte Wildpflanze auch den bayrischen Volksnamen «Bauchwehbleaml» (Bauchwehblümchen).

Laut neuesten Forschungen enthält das Edelweiss antibakterielle, entzündungshemmende sowie antioxidative Stoffe, deren Wirkstoffgehalt mit steigender Höhe des Standorts steigt. In der Volksheilkunde wird es heute noch zur Wundpflege eingesetzt. Zur Pflege sensibler Haut sowie als Sonnenschutz ist es auch bei Naturkosmetikfirmen sehr beliebt [2, 4].

\section{Schafgarbe (Achillea millefolium)}

Die Schafgarbe (Abb. 5) ist eine oft vergessene Heilpflanze in der Wundpflege, auch wenn ihr Name Achillea direkt mit ihrer Fähigkeit, Wunden zu heilen, verbunden ist. "Achillea» bezieht sich auf den griechischen Helden «Achilles», der in der Schlacht um Troja von Paris mit einem vergifteten Pfeil verwundet wurde. Chiron, der heilkundige Zentaur, soll ihm die Schafgarbe zur Heilung gereicht haben. Der deutsche Name «Garbe» kommt vom mittelhochdeutschen Wort «Garwe», was mit "Gesundmacher» übersetzt wird, und bezieht sich auf die breite Anwendung der Schafgarbe in den verschiedensten Indikationsgebieten (Leber-, Gallenund Magenbeschwerden, Frauenheilkunde, Herz-Kreislauf-Erkrankungen usw.). Andere volkstümliche Namen sind «Blutstillkraut», «Zimmermannskraut» und «Heil aller Schäden» [2]. 
Als wichtigste Inhaltsstoffe gelten ätherische Öle mit Proazulen, Gerbstoffe, Bitterstoffe und Flavonoide. Die Schafgarbe wird in der Wundheilkunde wegen ihrer wundheilenden, keimhemmenden, blutstillenden und entzündungshemmenden Wirkungen erfolgreich eingesetzt.

A. millefolium gehört wie die Ringelblume zur Familie der Korbblütler (Compositae). Bei Überempfindlichkeit auf Korbblütler sollte sie nicht verwendet werden $[2,4,6]$.

\section{Literatur}

1 Rippe O, Madejsky M: Die Kräuterkunde des Paracelsus. München, AT, 2006.

2 Bühring U: Praxis-Lehrbuch der modernen Heilpflanzenkunde. Stuttgart, Sonntag, 2005.

3 Kalbermatten R: Wesen und Signatur der Heilpflanzen. Aarau, AT, 2002.

4 Schramm H: Heilmittel der anthroposophischen Medizin. Elsevier, München, 2009.

\section{Spitzwegerich (Plantago lanceolata)}

Die Blätter des Spitzwegerich (Plantaginis lanceolatae folium) wie auch der anderen Wegerich-Arten sind gerade unterwegs - als erste Hilfe für Wunden, Blasen und Insektenstiche bekannt und beliebt. Ihre Wirksamkeit beruht auf ihrem Gehalt an Iridoidglykosiden (Aucubin, Catalpol usw.), Schleimstoffen, Gerbstoffen, Kieselsäure, Flavonoiden sowie Kaffeesäure- und Zimtsäurederivaten.
Pfarrer Künzle soll auf die Frage, ob bei äusserer Anwendung nicht die Gefahr einer Infektion bestehe, gesagt haben: «Das kennt der Spitzwegerich nicht. Ein solcher Verband ist der erste, manchmal der beste Notverband, denn die Heilung solcher Wunden geht rasch vor sich. Wie mit Goldfäden näht der Wegerichsaft den klaffenden Riss» [2]. P. lanceolata war die Arzneipflanze des Jahres 2014 und wurde in Ausgabe 1/2014 der Schweizerischen ZEITSCHRIFT FÜR GANZHEITSMEDIZIN ausführlich vorgestellt [9].
5 Schaffner W, Häfelfinger B, Ernst B: Heilpflanzen-Kompendium. Bern, Arboris, 1996.

6 www.smgp.ch.

7 Ganz C: Arzneipflanze des Jahres 2015: Johanniskraut (Hypericum perforatum). Schweiz Z Ganzheitsmed 2015;27:27-29.
Hunziker T, Hafner J, Streit M, Läculi S: Pflanzlicher Wundspray für akute und chronische Hautwunden. Wund Management 06/2012:270-274.

9 Ganz C: Arzneipflanze des Jahres 2014: Spitzwegerich (Plantago lanceolata). Schweiz Z Ganzheitsmed 2014;26:17-18. 